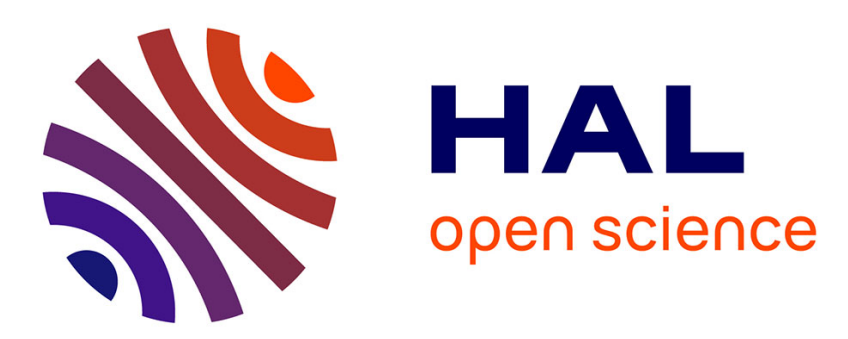

\title{
Influence de la congélation et de la cryo-dessiccation sur le taux de survie et le pourcentage des ferments lactiques dans le bioghurt
}

\author{
A. Jabarit
}

\section{To cite this version:}

A. Jabarit. Influence de la congélation et de la cryo-dessiccation sur le taux de survie et le pourcentage des ferments lactiques dans le bioghurt. Le Lait, 1969, 49 (488), pp.520-532. hal-00928500

\author{
HAL Id: hal-00928500 \\ https://hal.science/hal-00928500
}

Submitted on 1 Jan 1969

HAL is a multi-disciplinary open access archive for the deposit and dissemination of scientific research documents, whether they are published or not. The documents may come from teaching and research institutions in France or abroad, or from public or private research centers.
L'archive ouverte pluridisciplinaire HAL, est destinée au dépôt et à la diffusion de documents scientifiques de niveau recherche, publiés ou non, émanant des établissements d'enseignement et de recherche français ou étrangers, des laboratoires publics ou privés. 


\title{
Influence de la congélation et de la cryo-dessiccation sur le taux de survie et le pourcentage des ferments lactiques dans le bioghurt
}

\author{
par \\ A. JABARIT \\ Chef $d u$ Laboratoire \\ SEPIAL, Centre de Recherches et d'essais (92) Clichy
}

\section{INTRODUCTION}

Cette étude fait suite à notre travail paru précédemment dans la revue "Le lait " (1969, no 483-484, p. 160).

Nous y étudions l'influence des températures basses $\left(-20^{\circ} \mathrm{C}\right.$, $-25^{\circ} \mathrm{C},-30^{\circ} \mathrm{C},-35^{\circ} \mathrm{C},-40^{\circ} \mathrm{C}$ ) et la cryo-dessiccation qui s'ensuit sur la survie et le comportement des ferments lactiques mentionnés ci-dessous :

- Streptococcus Lactis,

- Lactobacillus acidophilus,

au lieu du Streptococcus thermophilus et du Lactobacillus bulgaricus. Ces deux ferments lactiques n'ont pas de vie symbiose comme c'est le cas pour le yaourt ordinaire.

En réalité, c'est un mélange des deux ferments non symbiotiques. Néanmoins, le produit du métabolisme coagule exactement le lait comme les ferments lactiques du yaourt classique. Celui-ci a un goût spécial, plutôt crémeux, mais son aspect général est exactement le même que celui du yaourt ordinaire. C'est pourquoi nous l'appelons Bioghurt. D'ailleurs dans certains pays, notamment la Suisse, il est connu sous cette appellation.

Ce travail est toujours réalisé dans le cadre d'une action concertée entre SOCALTRA et D.G.R.S.T., contrant $\mathrm{n}^{\circ}$ 68-01-320-00-212-75-01.

\section{PROTOCOLE D'ESSAI}

Le protocole utilisé est rigoureusement semblable aux essais précédents, à savoir :

- Préparer et stériliser du lait destiné à la fabrication du bioghurt 
dont sa composition reste toujours la même :

- lait écrémé en poudre : $140 \mathrm{~g}$,

- eau distillée q.s.p. : $1000 \mathrm{ml}$,

- le $\mathrm{pH}: 6,5$.

- Ensemencer à 5 p. 100 avec du levain connu dans notre laboratoire :

- Streptococcus lactis,

- Lactobacillus acidophilus.

- Etuver à $43^{\circ} \mathrm{C}$ pendant $2 \mathrm{~h}$. Refroidissement progressif jusqu'à la phase exponentielle.

- Prélever et contrôler :

- le nombre des ferments lactiques revivifiables par $\mathrm{ml}$, selon la méthode Hoskins (nombre le plus probable),

- le pourcentage des ferments lactiques par la méthode directe d'observation microscopique (frottis coloré au bleu de méthylène et examen à l'état frais avec une cellule Malassez et Vignal),

- le $\mathrm{pH}$,

- l'acidité en degré Dornic.

\section{Congélation et conservation}

En rapport avec notre série d'essais, contrôles systématiques et identiques par rapport à ceux du bioghurt frais, le temps de congélation aux températures basses étudiées varie entre 1 h. 15 et 2 h 45 (tableau).

La durée de conservation varie entre 12 h 15 et 13 h 45 .

Temps de congélation et de conservation du bioghurt suivant la température de congélation

\begin{tabular}{c|c|c|c|c}
\hline $\begin{array}{c}\text { Température } \\
\text { de } \\
\text { congélation }\end{array}$ & $\begin{array}{c}\text { Temps de } \\
\text { congélation } \\
\text { en h }\end{array}$ & $\begin{array}{c}\text { Durée de } \\
\text { conservation } \\
\text { en h }\end{array}$ & $\begin{array}{c}\text { Total } \\
\text { en h }\end{array}$ & Observations \\
\cline { 2 - 5 }$-20^{\circ} \mathrm{C}$ & $2 \mathrm{~h} 45$ & $12 \mathrm{~h} 15$ & $15 \mathrm{~h}$ & \\
$-25^{\circ} \mathrm{C}$ & $2 \mathrm{~h} 15$ & $12 \mathrm{~h} 45$ & $15 \mathrm{~h}$ & \\
$-30^{\circ} \mathrm{C}$ & $1 \mathrm{~h} 45$ & $13 \mathrm{~h} 15$ & $15 \mathrm{~h}$ & \\
$-35^{\circ} \mathrm{C}$ & $1 \mathrm{~h} 30$ & $13 \mathrm{~h} 30$ & $15 \mathrm{~h}$ & \\
$-40^{\circ} \mathrm{C}$ & $1 \mathrm{~h} 15$ & $13 \mathrm{~h} 45$ & $15 \mathrm{~h}$ & \\
\hline
\end{tabular}




\section{Détermination de la charge optimale}

Nous avons conservé la charge $1,8 \mathrm{~kg}$ soit $8 \mathrm{~kg} / \mathrm{m}^{2}$ qui correspond à notre possibilité de travail journalier.

\section{Durée du cycle}

La durée du cycle de la cryo-dessiccation varie entre 8 et 9 h.

\section{Cryo-dessiccation du bioghurt}

La cryo-dessiccation du bioghurt s'effectue suivant différentes phases selon la nature de l'eau contenue dans le bioghurt. Dans le bioghurt, l'eau est répartie sous plusieurs formes (5) :

- eau liée ou eau d'hydratation des protéines,

- eau immobilisée ou eau capillaire,

- eau libre ou mobile en sérum,

- eau vitale des ferments lactiques et eau non cristallisable des complexes lipido-protidiques (3).

Voici les différentes phases de sublimation :

a) Première phase : l'eau de sérum et l'eau capillaire (dessiccation primaire).

b) Deuxième phase : eau liée ou eau d'hydratation des protéines (dessiccation secondaire).

c) Troisième phase : eau vitale des ferments lactiques et eau non cristallisable des complexes lipido-protidiques (dessiccation tertiaire).

D'autre part dans le graphique $\mathrm{n}^{\circ} 4$, nous avons présenté des exemples de cryo-dessiccation avec ses différentes phases.

\section{Détermination de la fin de la cryo-dessiccation ou de l'humidité résiduelle}

Cette étape du processus de la cryo-dessiccation est extrêmement importante, en particulier sur le nombre des ferments lactiques revivifiables et leur survie au cours du vieillissement.

A la fin de la dessiccation primaire, le pourcentage de l'humidité résiduelle est supérieure à 8 p. 100, au cours de la dessiccation secondaire, l'humidité résiduelle peut varier entre 4 et 7 p. 100 . Pendant la dessiccation tertiaire, le pourcentage de l'humidité varie entre 4 et 1 p. 100, voire au-dessous de 1 p. 100 , ce qui risque de provoquer la réaction de Maillard (8).

Vers la fin du cycle, l'écart d'échange thermique est négligeable, les températures basses et hautes s'alignent à la température de consigne prévue à l'avance sur la surface du bioghurt. Par expérience, nous avons constaté que la température optimale de surface 
pour celui-ci est de $35^{\circ} \mathrm{C}$. Au-delà, le risque de brunissement et même de caramélisation est en cause (4).

Le bioghurt cryo-desséché est mis en bocaux de verre ou en sachets " complexe polyéthylène-aluminium-polypropylène ", sous vide et compensé à l'azote. Le temps de mise en emballage, dans ce cas " bioghurt " n'est pas limité, car le produit cryo-desséché n'est pas hygroscopique.

L'emballage sous atmosphère contrôlée est superflu.

\section{Prélèvement et analyses du bioghurt cryo-desséché}

Les analyses suivantes sont systématiquement effectuées :

- analyses physico-chimiques,

- analyses biochimiques et bactériologiques,

- analyses sensorielles et organoleptiques.

\section{Identification des micro-organismes réactifs utilisés}

1) Origine

Il s'agit des ferments lactiques :

- Streptococcus lactis du 31-10-56 X 13,

- Lactobacillus acidophilus du 9-7-57-3 009,

provenant de l'Ecole Polytechnique Fédérale de Zurich, régénérés, isolés et conservés dans notre laboratoire.

2) Aspect microscopique

Les ferments lactiques sont:

- Streptococcus lactis (cellules rondes, régulières, d'une dimension de 2 à 3 microns environ, présentés toujouss deux par deux,

- Lactobacillus acidophilus (bâtonnets longs de 5 à 6 microns). Ces ferments lactiques ont une coloration Gram positif.

\section{Caractéristiques taxonomiques}

Streptococcus lactis : c'est un streptocoque homo-fermentatif très répandu dans la nature.

Il croît même à la température inférieure à $10^{\circ} \mathrm{C}$ et à $40^{\circ} \mathrm{C}$, mais non pas à $45^{\circ} \mathrm{C}$. Il fait virer le tournesol rapidement et complètement avant d'avoir caillé le lait. D'après Sherman ( 9 et 10) et Abd-El-Malek et Gibson (1). La croissance a lieu en présence de 4 p. 100 de chlorure de sodium mais non de 6,5 p. 100 et à un pH de 9,2 et non de 9,6 , tandis que de l'ammoniac est produit à partir de l'arginine et de la peptone. Il produit de l'acide lactique $L(+)(7)$. 
Voici la galerie d'identification des caractères biochimiques du Streptococcus lactis :

- Gram + = cocci de diamètre de 2 à 3 microns.

- Formation de capsule : -

- Lait tournesolé $=$ réduction rapide du tournesol (acidité).

- Culture à $10^{\circ} \mathrm{C}=+$.

- Culture à $37^{\circ} \mathrm{C}=+$.

- Culture à $39^{\circ} \mathrm{C}=+$.

- Culture à $45^{\circ} \mathrm{C}=$ - .

- Hydrolyse de l'arginine $=+$.

- Culture avec 3 p. 100 de $\mathrm{Nacl}= \pm$.

- Culture avec 6,5 p. 100 de $\mathrm{Na} \mathrm{cl}=$ -

- Culture avec 0,0005 p. 100 de bleu de méthylène + lent.

- Synthèse du Dextrane $=$ -

- $\mathrm{pH}$ final dans le bouillon $=4,5-5$.

- Résistance à $55^{\circ} \mathrm{C} 15 \mathrm{mn}=+$.

- Résistance à $55^{\circ} \mathrm{C} 30 \mathrm{mn}=+$.

- Fermentation du glucose $=+$.

- Fermentation de l'arabinose $=+$ rare.

- Fermentation du xylose $=$ - .

- Fermentation du fructose $= \pm$.

- Fermentation du galactose $=+$.

Milieu Elliker (2), pour Streptococcus lactis

Voici la formule :

- Extrait de levure Difco . . . . . . . . . . . . . . . . . . . . 5 g

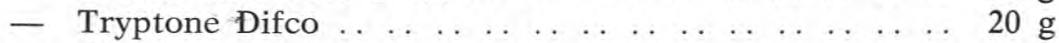



- Lactose .. . . . . . . . . . . . . . . . . . . . . . . . . . . . . . 5 g g

— Chlorure de sodium . . . . . . . . . . . . . . . . . . . . 15 g

- Eau distillée . . . . . . . . . . . . . . . . . . . . . . . . . . . $1000 \mathrm{ml}$

- $\mathrm{pH}=7,0$.

$\mathrm{pH}=7,0:$ nombre des cellules revivifiables $9 \times 10^{\circ} / \mathrm{ml}$.

$\mathrm{pH}=6,5:$ nombre des cellules revivifiables 2,5 à $4 \times 10^{10}$ (phase exponentielle).

Lactobacillus acidophilus : c'est un micro-organisme thermophile. Il a été isolé pour la première fois à partir de faeces d'enfants nourris au lait. Bien qu'il ressemble au Lactobacillus Bulgaricus, il existe des différences taxonomiques très nettes entre ces deux espèces. Le Lactobacillus acidophilus produit de l'acide lactique DL, et fermente une gamme plus étendue de sucres. De plus, il est très résistant à la bile.

Voici la galerie d'identification du Lactobacillus acidophilus :

- Culture à $15^{\circ} \mathrm{C}=-$.

- Culture à $45^{\circ} \mathrm{C}=+$.

- Résistance à $60^{\circ} \mathrm{C} / 90 \mathrm{mn}=$ -

- Résistance à $65^{\circ} \mathrm{C} / 30 \mathrm{mn}=-$. 
- Acide p. 100 dans le lait : 0,8.

- Configuration de l'acide lactique $=\mathrm{DL}$.

- Production du gaz à partir du sucre $=-$.

- Production d'NH $\mathrm{NH}_{3}$ à partir de l'arginine $=-$.

- Culture en Nacl 2 p. $100=+$.

- Culture en Nacl 4 p. $100=+$.

$\mathrm{R}$ : Riboflavine

- Exigences nutritionnelles $=$ RFC $\quad \mathrm{F}$ : Acide Folique

C : Cyanocobalamine

- Fermentation des sucres : arabinose $=-$.

- Fermentation des sucres comme :

$$
\text { xylose }=- \text {. }
$$

- le glucose +

- le galactose +

- le lactose +

- le maltose $\quad+$

- le salicine +

- le saccharose +

- le treahalose +

- L'hydrolyse de l'esculine $=$ -

- La croissance avec 1 p. 100 de bile $+=+$.

- La croissance avec 3 p. 100 de bile $=+$.

Il s'agit ici du pourcentage de bile liquide et non de bile sêche ou de sels biliaires. La bile employée était une solution à $10 \mathrm{p} .100$ de bile de bovin séchée (oxoïd). Le milieu à 1 p. 100 de bile contient donc 0,1 p. 100 de bile sèche (6).

Le Lactobacillus acidophilus est biochimiquement bien distinct, à savoir :

- L'acidification dans le lait est faible, de nombreux sucres différents peuvent être assimilés. Cette espèce est la seule à pouvoir s'implanter parmi la flore intestinale des enfants nourris au biberon : on la trouve souvent aussi dans les faeces de l'homme adulte et des mammifères. La possibilité de se multiplier dans l'intestin, pour le Lactobacillus acidophilus est très intéressante.

Influence des températures basses et de la cryo-dessiccation sur le taux de deux ferments lactiques revivifiables:

- Streptococcus lactis,

- Lactobacillus acidophilus,

dans le bioghurt avant la phase exponentielle.

1) On constate le seuil de sensibilité des ferments lactiques qui se situe à partir de $-20^{\circ} \mathrm{C}$.

2) La perte des ferments lactiques se stabilise à la température de congélation à partir de $-30^{\circ} \mathrm{C}$.

3) Le graphique $n^{\circ} 1$ a été établi sur la moyenne de 4 courbes pour chaque température de congélation. 




Température de congélation en degrés centigrades congelés cryo-desséchés

Influence des températures basses et de la cryo-dessiccation qui s'ensuit sur le pourcentage de deux ferments lactiques revivifiables :

- Streptococcus lactis,

- Lactobacillus acidophilus,

dans le bioghurt avant la phase stationnaire.

GRAPHIQUE $N^{\circ} 2$

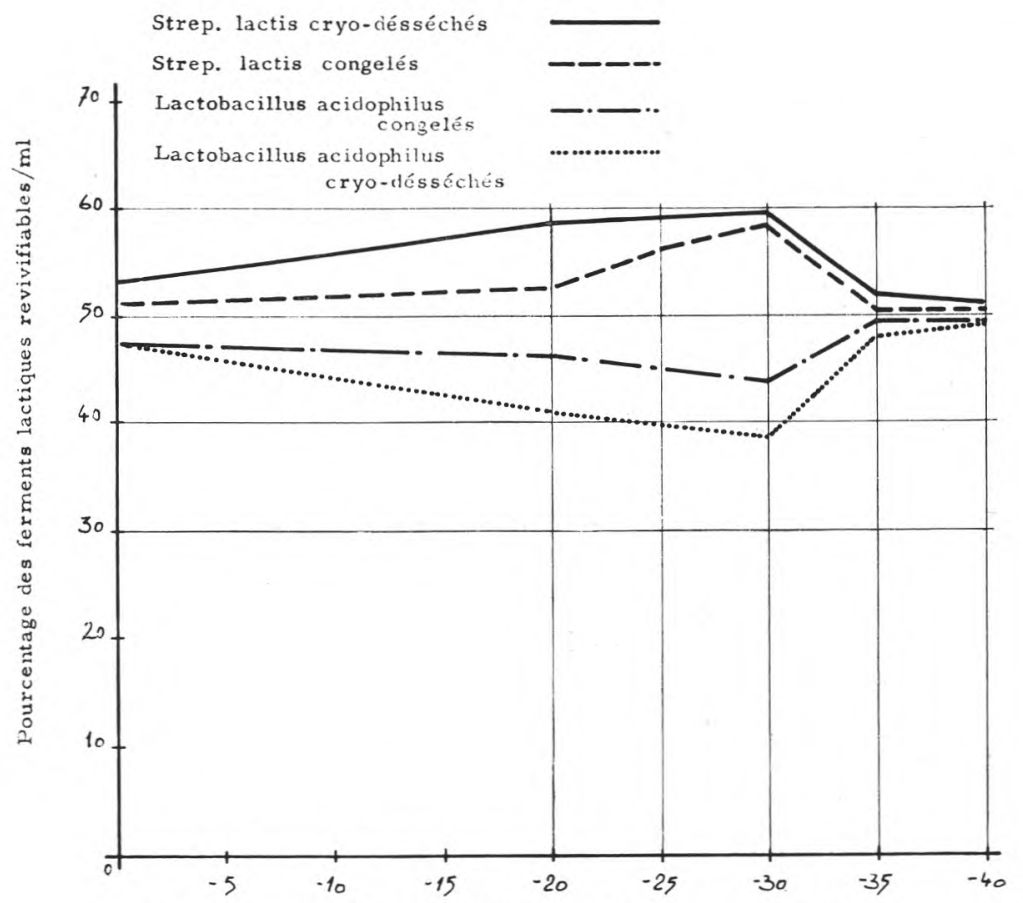

Température de congélation en degrés centigrades 
1) On constate que la disproportion entre les deux ferments lactiques commence à partir de la température de congélation de $-20^{\circ} \mathrm{C}$.

2) La disproportion s'accentue au maximum à la température de congélation de $-30^{\circ} \mathrm{C}$.

3) La disproportion du pourcentage respectif des ferments lactiques présents redevient négligeable à partir de la température de congélation de $-35^{\circ} \mathrm{C}$.

4) La disproportion du pourcentage respectif s'accentue à la cryo-dessiccation qui s'ensuit jusqu'à la température de congélation de $-35^{\circ} \mathrm{C}$.

5) Ce graphique a été établi sur la moyenne de 4 courbes correspondant pour chaque température de congélation.

GRAPHIQUE N० 3

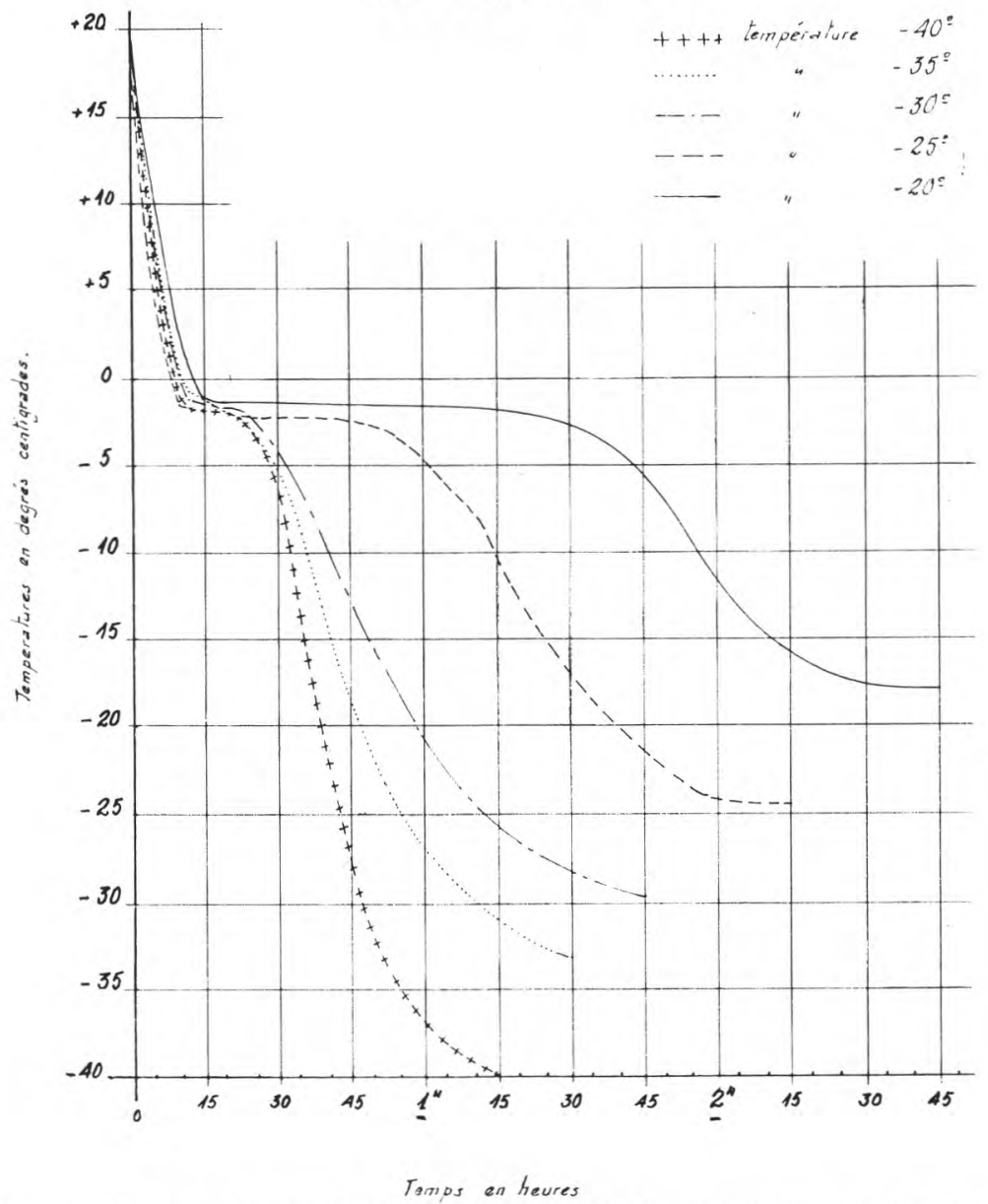

Influence des températures basses sur la durée de congélation du bioghurt 
GRAPHIQUE $N^{\circ} 4$

Cryo-dessiccation du bioghurt avec ses différentes phases

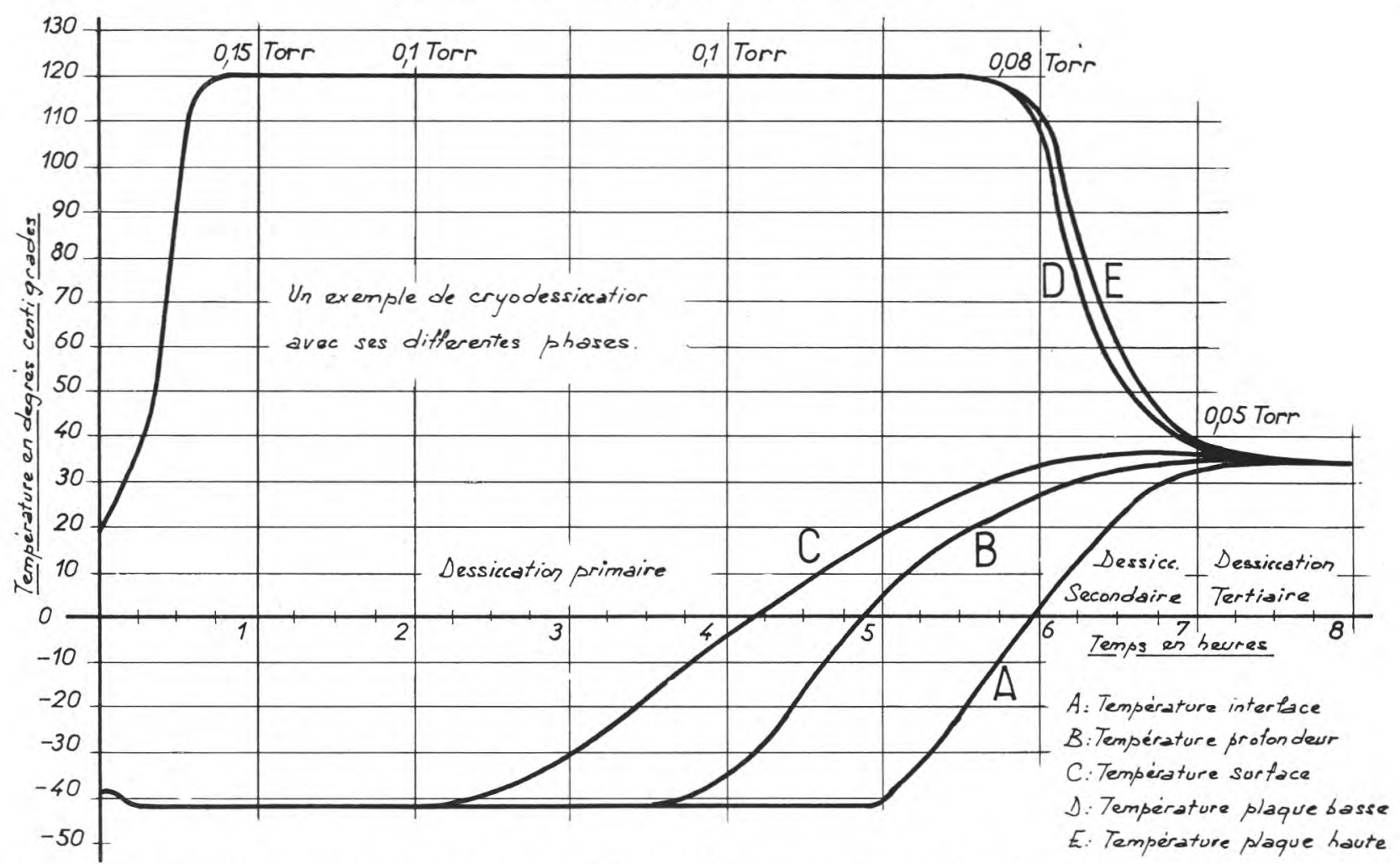

Dessiccation primaire : eau de sérum et eau capillaire - H.R. est supérieure à 8 p. 100 .

Dessiccation secondaire : eau liée ou eau d'hydratation des protéines - H.R. varie entre 4 à 7 p. 100 .

Dessiccation tertiaire : eau vitale des ferments lactiques et eau non cristallisable des complexes lipido-protidiques H.R. varie entre 1 à 2 p. 100 (voire au-dessous de 1 p. 100).

Il existe une relation directe entre la pression de la vapeur et la température de chauffage pour chaque phase de la cryo-dessiccation.

H.R. : Humidité résiduelle. 


\section{Description du mode opératoire}

- Plateau SEPIAL ayant les dimensions suivantes :

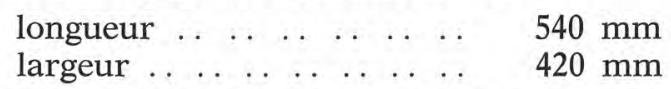

- La prise d'essai «bioghurt » est de $1,8 \mathrm{~kg}$, soit $10 \mathrm{~mm}$ d'épaisseur de produit à congeler.

- Les thermocouples sont fixés à différentes profondeurs du produit et reliés à l'enregistreur automatique.

- L'échange thermique dans le congélateur s'effectue du bas vers le haut ; la température de congélation est réglable par un thermostat de $-20^{\circ} \mathrm{C}$ à $-40^{\circ} \mathrm{C}$ avec une précision de $\pm 1^{\circ} \mathrm{C}$.

Nous avons constaté que le temps de formation des micro-cristaux et la prise en masse totale varie entre $1 \mathrm{~h} 15$ et $2 \mathrm{~h} 45$, suivant la température de congélation. Nous avons donc basé notre calcul de la vitesse de congélation sur l'épaisseur de $10 \mathrm{~mm}$ du bioghurt. Celle-ci varie suivant la température de congélation entre $3 \mathrm{~mm}$ à $8 \mathrm{~mm} / \mathrm{h}$.

Vitesse de la congélation du bioghurt suivant la température de congélation

\begin{tabular}{c|c|c|c}
\hline $\begin{array}{c}\text { Températures } \\
\text { de } \\
\text { congélation }\end{array}$ & $\begin{array}{c}\text { Temps de } \\
\text { congélation } \\
\text { en h }\end{array}$ & $\begin{array}{c}\text { Vitesse de } \\
\text { congélation }\end{array}$ & Observations \\
\cline { 2 - 3 }$-20^{\circ} \mathrm{C}$ & $2 \mathrm{~h} 45$ & $3 \mathrm{~mm} / \mathrm{h}$ & \\
$-25^{\circ} \mathrm{C}$ & $2 \mathrm{~h} 15$ & $4 \mathrm{~mm} / \mathrm{h}$ & \\
$-30^{\circ} \mathrm{C}$ & $1 \mathrm{~h} 45$ & $5 \mathrm{~mm} / \mathrm{h}$ & \\
$-35^{\circ} \mathrm{C}$ & $1 \mathrm{~h} 30$ & $6,5 \mathrm{~mm} / \mathrm{h}$ & \\
$-40^{\circ} \mathrm{C}$ & $1 \mathrm{~h} 45$ & $8 \mathrm{~mm} / \mathrm{h}$ & \\
\hline
\end{tabular}

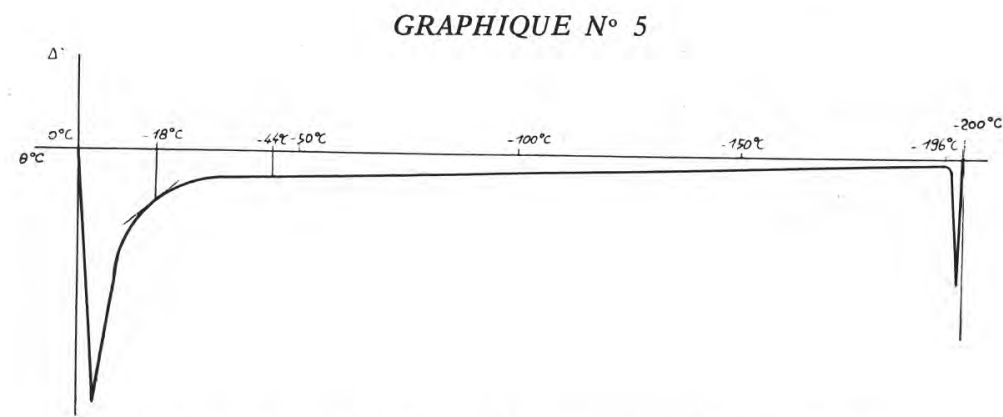

Température de fusion commençante du bioghurt

"Analyse thermique différentielle du bioghurt " 


\section{Conclusions bioghurt}

Pour ces expériences, nous avons choisi deux ferments lactiques:

- Streptococcus lactis,

- Lactobacillus acidophilus,

pour étudier le comportement et la viabilité de ceux-ci aux températures de congélation.

En effet, nous avons obtenu des résultats reproductibles prouvant la sensibilité des ferments lactiques étudiés, en fonction des températures de congélation, à savoir :

- le froid exerce un effet important sur la viabilité de nos ferments en particulier les Lactobacillus acidophilus subissent une perte énorme, de sorte que la disproportion entre les deux ferments varie entre 5 et 20 p. 100, suivant la température de congélation, - nous pouvons classer quantitativement les influences des températures basses, en plusieurs catégories :

a) La température de congélation à $-20^{\circ} \mathrm{C}$ n'a pas un rôle considérable sur le taux de deux ferments lactiques revivifiables.

b) La température de $-25^{\circ} \mathrm{C}$ a un rôle nuisible chiffrable sur le pourcentage respectif; cette disproportion est de l'ordre de 5 p. 100 au détriment des Lactobacillus acidophilus.

c) La température de congélation de $-30^{\circ} \mathrm{C}$ a un rôle destructif sur la viabilité de deux ferments lactiques et leur pourcentage respectif ; celui-ci présente un chiffre de 20 p. 100 en faveur des Streptococcus lactis.

L'écart du pourcentage respectif des ferments lactiques présents commence à partir de la température de congélation de $-25^{\circ} \mathrm{C}$ et celui-ci s'accentue à la température de congélation de $-30^{\circ} \mathrm{C}$. Cependant l'écart du pourcentage s'affaiblit à partir de $-35^{\circ} \mathrm{C}$ de sorte que celui-ci est négligeable à $-40^{\circ} \mathrm{C}$.

Au cours de la cryo-dessiccation, cet écart du pourcentage entre les Streptococcus lactis et les Lactobacillus acidophilus, s'accentue parallèlement par rapport à la température de congélation, mais à partir de $-35^{\circ} \mathrm{C}$, cet écart devient négligeable.

On peut en déduire que l'effet du froid sur le taux et le pourcentage respectif des ferments lactiques présents est important et la cryo-dessiccation n'intervient pas sur la disproportion de deux ferments lactiques.

De toute évidence, il en ressort que les Lactobacillus acidophilus sont très sensibles au froid. Il faudrait donc continuer à chercher d'autres souches plus résistantes aux températures de congélation.

Enfin, nous avons constaté que la température optimale de la surface du bioghurt cryo-desséché pendant la $3^{\text {me }}$ phase du séchage (adsorption ou dessication tertiaire) se situe entre $25^{\circ} \mathrm{C}$ et $35^{\circ} \mathrm{C}$, car elle permet de réduire le temps de dessication tertiaire. Celle-ci a un rôle efficace sur la viabilité des ferments lactiques cryo-desséchés. 


\section{Remerciements}

Nous remercions $M$. le Professeur Thieulin pour les précieux conseils qu'il a bien voulu nous donner pour la rédaction de cet article.

Nous remercions également les Directions Générales de SOCALTRA et de SEPIAL, ainsi que tous les membres du Centre de Recherche qui nous ont aidés pour la réalisation de ce travail.

Nous exprimons nos remerciements à la Délégation générale à la Recherche Scientifique et Technique de nous avoir confié la réalisation de ce travail.

\section{R és u m é}

En définitive, on peut en déduire que l'effet du froid sur le taux et le pourcentage respectif des ferments lactiques présents est important et la cryo-dessiccation n'intervient pas sur la disproportion de deux ferments lactiques.

De toute évidence, il en ressort que les Lactobacillus acidophilus sont très sensibles au froid. Il faudrait donc continuer à chercher d'autres souches plus résistantes aux températures de congélation.

Enfin, nous avons constaté que la température optimale de la surface du bioghurt cryo-desséché pendant la $3^{\text {me }}$ phase du séchage (adsorption ou dessiccation tertiaire) se situe entre $25^{\circ} \mathrm{C}$ et $35^{\circ} \mathrm{C}$, car elle permet de réduire le temps de dessiccation tertiaire. Celle-ci a un rôle efficace sur la viabilité des ferments lactiques cryo-desséchés.

\section{Su m m a ry}

Finaly, we may make this conclusion : the freezing has an important influence on viability and percentage between the lactic acid bacteria. But, freeze-drying has not a role on disproportion between these lactic acid bacteria.

Anyhow, it is evident that «Lactobacillus acidophilus » is sensible to freezing. We shall continue our research on others strains wich are much more resistant to freezing temperatures.

We find also, that the best surface temperature for Bioghurt (Str lactis and Lb acidophilus) during third phase of dessiccation is situed between $25^{\circ} \mathrm{C}$ and $35^{\circ} \mathrm{C}$. Because, it is important for lactic acid bacteria viability and to make shorter the freeze-drying cycle. 


\section{Bibliographie}

[1] AbD-el-Malek (J.) et Gibson (T.). - J. Dairy Research, 15 (1948), p. 233.

[2] Elliker (P. R.), Anderson (A. M.) and Handesson (G.). - Anagar medium for lactic acid streptococci and bacto bacilli. J. Dairy Sc., 39 (1956), p. $1611-1612$.

[3] JabaRit (A.). - Influence de la congélation et de la cryo-dessiccation sur le taux de survie et le pourcentage des ferments lactiques dans le yoghourt, Le Lait (1969), $\mathrm{n}^{\circ} 483-484$, p. 160.

[4] Kuprianoff (J.). - Freeze drying of foods nat. Ac. Sc. Nat. Res. Couvial (Washington D.C.) (1962), p. 24.

[5] Kurmann (A.). - Etudes biotechniques sur la fermentation du yoghourt doux et aromatique. Le Lait, 47 (1967), 267, p. 415.

[6] Mocouot (G.). - L'industrie Latière, 159 (1959).

[7] ORLa (J. S.) - The lactic acid Bacteria, Copenhagen (1919 et 1943).

[8] Solwin (H.). - Freeze drying of foods. Nat. Ac. Res. Council - C. Washington D.C. (1962).

[9] Sherman (J. H.). - J. Dairy Sc., 38 (1955), p. 1184.

[10] Sherman (J. M.). - Bacteria Rev. (1937), I.I. 